\title{
SPECTRAL FUNCTIONS OF THE QUANTUM DOT COUPLED TO NORMAL AND/OR SUPERCONDUCTING LEADS
}

\author{
M. KRAWIEC AND K.I. WYSOKIŃSKI \\ Institute of Physics, M. Curie-Skłodowska University \\ Radziszewskiego 10, 20-031 Lublin, Poland
}

\begin{abstract}
We study the spectrum of the quantum dot coupled to two external leads, which may be normal and/or superconducting. The dot is described by the Anderson-Hubbard impurity model in the infinity $U$ limit. The impurity spectral function shows complex behavior depending on the temperature and the state of the leads. For normal leads and at low temperature we observe the appearance of the Kondo resonance, while for BCS-like superconducting leads the bound states emerge due to absence of low energy excitations in the leads.
\end{abstract}

PACS numbers: 73.40.Gk, 72.15.Qm, 71.27.+a

The study of the electric current flowing through the quantum dot coupled to two external leads has recently attracted much attention [1]. Experimentally one measures the current as a function of source-drain voltage $V_{\mathrm{SD}}$ at various temperatures and other parameters, as e.g. the coupling strength between dot and external electrodes. The experiments $[2,3]$ show that one observes the same physics as in metals containing magnetic impurities.

The current through the system is given by an energy integral [4]

$$
J=\frac{e}{\hbar} \sum_{\sigma} \int \mathrm{d} \omega\left[f_{\mathrm{L}}(\omega)-f_{\mathrm{R}}(\omega)\right] \frac{\Gamma_{\sigma}^{L}(\omega) \Gamma_{\sigma}^{R}(\omega)}{\Gamma_{\sigma}^{L}(\omega)+\Gamma_{\sigma}^{R}(\omega)}\left(\frac{-1}{\pi}\right) \Im G_{\sigma}\left(\omega \mid \mathrm{i} 0^{+}\right) .
$$

Here $f_{\lambda}(\omega)$ denotes the Fermi distribution function for lead $\lambda=\mathrm{L}$ (left) or $\mathrm{R}$ (right) with chemical potential $\mu_{\lambda}, G_{\sigma}(\omega)$ is the impurity Green function and $\Gamma^{\lambda}(\omega)=2 \pi \sum_{k}\left|V_{\lambda k}\right|^{2} \delta\left(\omega-\varepsilon_{\lambda k}\right)$ is the effective coupling of localized electron to conduction band (see below). The difference between the chemical potentials $\mu_{\mathrm{L}}-\mu_{\mathrm{R}}=e V_{\mathrm{SD}}$ is kept constant by the source-drain voltage.

As seen from (1) the current through the system requires the knowledge of the dot's spectral function $A(\omega)=-\frac{1}{\pi} \Im G(\omega+\mathrm{i} 0)$. It depends on the spectrum of electrons in the leads, the strength of coupling between dot and leads, position of the energy level in the dot and other details. It is the purpose of this work to study the spectral functions of the dot coupled to normal and/or superconducting leads with varying parameters. 
In the previous paper [5] we have shown that the presence of the Van Hove singularity near the Fermi level in the spectrum of electrons in the leads induces additional structure in $A(\omega)$ and differential conductance $G\left(V_{\mathrm{SD}}\right)=\mathrm{d} J / \mathrm{d} V_{\mathrm{SD}}$. Robustness of this structure against temperature changes distinguishes it from the more interesting Kondo peak.

To calculate $A(\omega)$ we start with the following model:

$$
\begin{aligned}
H= & \sum_{\lambda k \sigma} \varepsilon_{\lambda k} c_{\lambda k \sigma}^{+} c_{\lambda k \sigma}+E_{\mathrm{d}} \sum_{\sigma} d_{\sigma}^{+} d_{\sigma}+\frac{1}{2} U \sum_{\sigma} d_{\sigma}^{+} d_{\sigma} d_{-\sigma}^{+} d_{-\sigma} \\
& +\sum_{\lambda k \sigma}\left(V_{\lambda k} c_{\lambda k \sigma}^{+} d_{\sigma}+\text { h.c. }\right)+\sum_{i j} W_{i j} n_{i} n_{j} .
\end{aligned}
$$

The parameters have the following meaning: $c_{\lambda k \sigma}^{+}\left(c_{\lambda k \sigma}\right)$ denote creation (annihilation) operator for a conduction electron with wave vector $k$, spin $\sigma$ in the lead $\lambda, V_{\lambda k}$ is the hybridization matrix element between conduction electron of energy $\varepsilon_{\lambda k}$ in a lead $\lambda$ and electron localized on the dot. $E_{\mathrm{d}}$ is the single particle energy at the dot. Here $n_{i}=\sum_{\sigma} c_{i \sigma}^{+} c_{i \sigma}$ is the number operator for electrons in the electrodes, $W_{i j}$ is the electron-electron interaction energy which leads to $s$ or $d$-wave superconductivity in the electrodes, while $U$ is the (repulsive) interaction energy between two electrons localized on the dot. To calculate the on-dot Green function $G_{\sigma}(\omega)$ we used the modified slave boson technique in the $U=\infty$ limit $[6,7]$. For the purpose of numerical calculations we neglected the wave vector dependence of the hybridization and used $V_{\mathbf{L} k}=V_{\mathbf{R} k}=V$ and constant density of states for normal electrons in the leads. Thus $\Gamma(\omega)=\Gamma_{\mathrm{L}}=\Gamma_{\mathrm{R}}=2 \pi V^{2} / D$, where $D$ is the band width taken below as an energy unit.

In Fig. 1 we show the density of states (DOS) of the quantum dot coupled to two normal leads. The chemical potentials $\left(\mu_{\mathrm{L}}=-0.05\right.$ and $\left.\mu_{\mathrm{R}}=0.05\right)$ are shifted from their common position by the applied external source-drain voltage. Different curves correspond to different positions of the on-dot energy level $E_{\mathrm{d}}$ (located below, between, and above the chemical potentials). Note the appearance of the Kondo peak each time the energy $E_{\mathrm{d}}$ lies below the chemical potential and the "Kondo dip" for $E_{\mathrm{d}}$ above it.

If the dot is coupled to two BCS-like superconductors with constant superconducting gap $\Delta$, then there is no Kondo resonance in the DOS. Instead we observe the appearance of two bound states at energies falling inside superconducting gap. This behavior is shown in Fig. 2. We have plotted DOS in the energy range close to the Fermi level of the system. The solid line corresponds to equilibrium situation $\left(\mu_{\mathrm{L}}=\mu_{\mathrm{R}}=0\right)$ while the dashed one for $\mu_{\mathrm{L}}=-\mu_{\mathrm{R}}=-\Delta / 5$. Except bound states (as in equilibrium) there emerge additional structures in the regions, where one of the leads has a finite density of states.

Our model allows for a $d$-wave superconductivity in the electrodes. Figure 3 shows the spectral density of the dot connected to two external leads being $d$-wave superconductors. We note that the DOS vanishes linearly with energy. The additional structure seen in the curve is the remnant of the bound states, which have turned into resonances. If one of the leads is normal and other superconducting one finds that DOS contains both Kondo peak and resonant states. The Kondo peak can also be observed in systems with superconducting leads provided $V_{\lambda k}$ 

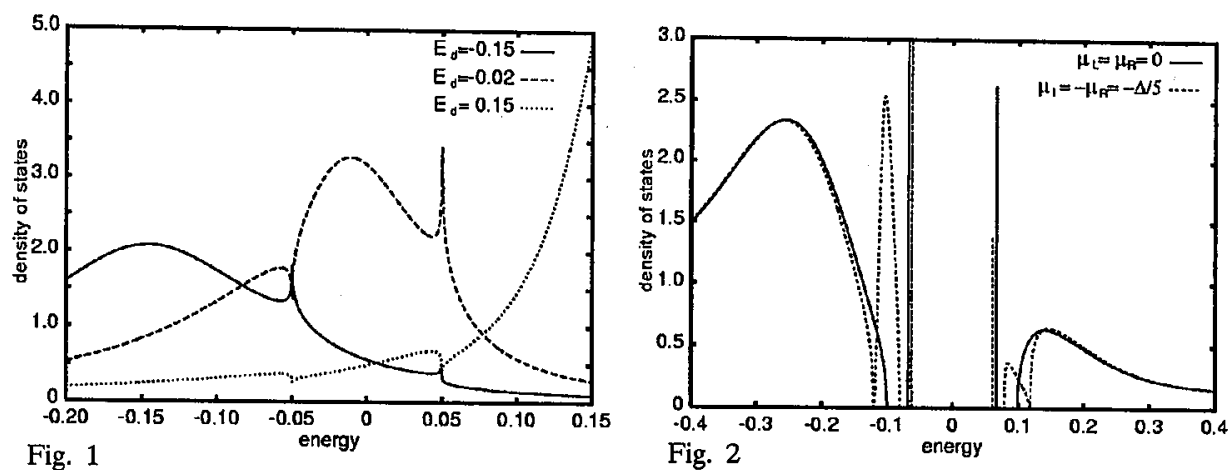

Fig. 1

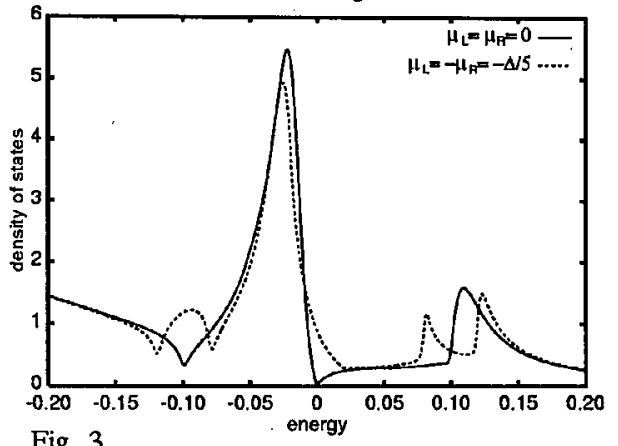

Fig. 3

Fig. 1. The density of states of quantum dot connected to two normal electrodes. The parameters are the following: the hybridization $V=0.1 D$, chemical potentials $\mu_{\mathrm{R}}=-\mu_{\mathrm{L}}=0.05 D$ and temperature $T=10^{-4} \mathrm{D}$.

Fig. 2. The density of states of quantum dot coupled to two superconducting electrodes. The energy $E_{\mathrm{d}}=-0.2 D, V=0.15 D$, and $s$-wave gap $\Delta=0.1 D$.

Fig. 3. The same as in Fig. 2 but electrodes are $d$-wave superconductors, with $\Delta(k)=$ $\Delta\left[\cos \left(k_{x} a\right)-\cos \left(k_{y} a\right)\right], \Delta=0.05 D, E_{\mathrm{d}}=-0.2 D$, and $V=0.15 D$.

exceeds some critical value. Similar conclusion has been obtained for system in which quantum dot was coupled to normal leads with DOS vanishing on the Fermi level [8].

In conclusion, we have studied the evolution of the density of states on the dot in dependence of the state (normal or superconducting) of external leads. One observes a variety of structures: Kondo peak, "Kondo dip", bound and resonant states, or combinations of them. The influence of these structures on the nonequilibrium transport is under study and will be published elsewhere.

\section{Acknowledgments}

This work has been partially supported by the Committee for Scientific Research under grant 2P03B 03111. 


\section{References}

[1] T.K. Ng, P.A. Lee, Phys. Rev. 61, 1768 (1989); L.I. Glazman, M.E. Raikh, Pis'ma Zh. Eksp. Teor. Fiz. 48, 378 (1988) [JETP Lett. 47, 452 (1988)]; S. Herschfield, J.H. Davies, J.W. Wilkins, Phys. Rev. Lett. 67, 3720 (1991).

[2] D. Goldhaber-Gordon, H. Shtrikman, D. Mahalu, D. Abusch-Magder, U. Meirav, M.A. Kastner, Nature 391, 1569 (1998).

[3] S.M. Cronenwett, S.M. Maurer, S.R. Pate, C.M. Marens, C.I. Durnöz, J.S. Harris, Jr., Phys. Rev. Lett. 81, 5904 (1998); S.M. Cronenwett, T.H. Oosterkamp, L.P. Konwenhoven, Science 281, 540 (1998).

[4] Y. Meir, N.S. Wingreen, P.A. Lee, Phys. Rev. Lett. 70, 2601 (1993); N.S. Wingreen, Y. Meir, Phys. Rev. B 49, 11040 (1994).

[5] M. Krawiec, T. Domański, K.I. Wysokiński, Acta Phys. Pol. A 94, 411 (1998).

[6] J.C. Le Guillou, E. Ragoucy, Phys. Rev. B 52, 2403 (1995).

[7] M. Krawiec, K.I. Wysokiński, Phys. Rev. B 59, 9500 (1999).

[8] D. Withoff, E. Fradkin, Phys. Rev. Lett. 64, 1835 (1990). 